\title{
Réflexions prospectives sur une protection juridique des réfugiés écologiques
}

\section{Christel Cournil et Pierre Mazzega}

\section{(2) OpenEdition \\ 12 Journals}

Édition électronique

URL : https://journals.openedition.org/remi/3541

DOI : 10.4000/remi.3541

ISSN : $1777-5418$

Éditeur

Université de Poitiers

\section{Édition imprimée}

Date de publication : 30 juin 2007

Pagination : 7-34

ISBN : 978-2-911627-44-6

ISSN : 0765-0752

\section{Référence électronique}

Christel Cournil et Pierre Mazzega, «Réflexions prospectives sur une protection juridique des réfugiés écologiques ", Revue européenne des migrations internationales [En ligne], vol. $23-n^{\circ} 1$ | 2007, mis en ligne le 30 juin 2010, consulté le 14 avril 2022. URL : http://journals.openedition.org/remi/3541 ; DOI : https://doi.org/10.4000/remi.3541

Ce document a été généré automatiquement le 14 avril 2022.

(c) Université de Poitiers 


\title{
Réflexions prospectives sur une protection juridique des réfugiés écologiques
}

\author{
Christel Cournil et Pierre Mazzega
}

1 Suivant un décompte de 1998, les catastrophes écologiques auraient conduit plus de 25 millions ${ }^{1}$ de personnes à quitter leurs habitations, leurs régions ou leurs pays (Julienne, 2002). Ces migrations forcées (Gonin et Lassailly-Jabob, 2002 ; Lassailly-Jacob, 2006) résultent de causes écologiques très variées, des catastrophes brutales d'origine naturelle ou technologique (séismes, cyclones et ondes de tempête, tsunamis, accidents industriels majeurs, etc.) aux catastrophes plus insidieuses, se développant sur un temps long (sécheresse, divers impacts de l'augmentation du niveau de la mer, désertification, etc. $)^{2}$.

Comment organiser une protection juridique adéquate pour les personnes qui fuient ces catastrophes? À l'heure actuelle aucun instrument juridique spécialisé n'existe pour garantir une assistance, un accueil ou des droits aux « réfugiés écologiques ${ }^{3}$. En effet, si les textes supranationaux (Kiss et Beurier, 2004) relatifs à l'environnement, aux réfugiés ou aux étrangers (Cournil, 2005) se sont multipliés ces dernières années avec l'internationalisation du droit, il n'en demeure pas moins que la problématique du refuge écologique n'est pas abordée explicitement dans ces textes. La protection internationale offerte par la Convention de Genève de $1951^{4}$ (relative au statut des réfugiés) n'offre aucune garantie pour les victimes d'une catastrophe écologique. La vocation de cette Convention est de protéger les personnes craignant avec raison de subir des persécutions politiques, religieuses, raciales, ethniques, etc. Aucun motif de l'article $1^{\text {er }} \mathrm{A}$ de la Convention de Genève ne prévoit une protection pour une cause écologique. De surcroît, les autres textes internationaux (notamment ceux relatifs aux droits de l'Homme ${ }^{5}$ ) ainsi que les jurisprudences rendues par leurs organes de contrôle ${ }^{6}$ n'apportent pas encore de garanties suffisantes pour protéger des réfugiés écologiques. Seules les initiatives isolées et marginales de certains pays ${ }^{7}$ ont timidement abordé cette question (Bouteillet-Paquet, 2002 : 181). De même, une lecture très extensive de la 
récente protection temporaire ${ }^{8}$ communautaire offerte par l'Union Européenne aux ressortissants extra-communautaires pourrait, en cas de grave crise, organiser un accueil sous condition en Europe. En somme, les instruments juridiques qui seraient susceptibles d'apporter une protection «par ricochet » sont très limités (Cournil, 2006).

3 Face à cette carence juridique, la doctrine française, spécialisée sur les questions relatives au droit de l'environnement et au droit international, s'est réunie à Limoges le 23 juin 2005 pour amorcer une réflexion pluridisciplinaire et prospective sur la nécessité d'un statut international pour le "réfugié écologique»". En effet, une protection juridique internationale, à l'image de celle offerte par la Convention de Genève relative aux réfugiés politiques, est séduisante, voire souhaitable pour envisager des solutions à la question globale du refuge écologique.

4 À partir de ces différents constats, nous proposons ici des analyses générales portant sur la pertinence d'une protection des réfugiés écologiques au plan international. De quelle nature doit être cette protection: assistance sur place? Aide humanitaire? Statut régissant des droits et libertés permettant de trouver refuge dans un autre pays? Accueil temporaire lorsque l'environnement d'origine présente une résilience suffisante? Face à ces multiples formes d'assistance a priori envisageables, quelle gouvernance faut-il organiser pour apporter une aide efficace aux réfugiés écologiques?

5 Avant de mener une analyse juridique sur la protection des réfugiés écologiques, la complexité du contexte sociétal et environnemental est soulignée notamment au travers des notions de risque, vulnérabilité et résilience. Nous nous attachons ensuite à évaluer les capacités ou potentialités des concepts et instruments juridiques actuels pour construire une ou des protections juridiques inédites, les instruments juridiques internationaux actuels étant limités, voire inadaptés, pour proposer une protection ou même une assistance internationale contraignante pour les réfugiés écologiques. Ainsi le droit est face à de nombreux défis qu'il devra relever pour imaginer une protection adéquate pour ce nouveau type de refuge.

\section{Le refuge écologique}

6 Du fait de la complexité des interactions entre les sociétés et leur environnement, la notion même de "refuge écologique » reste difficile à cerner de manière univoque (Gouguet, 2006). En effet la connaissance scientifique que nous avons de la dynamique des écosystèmes et de leurs évolutions possibles sous l'effet de perturbations, reste parcellaire et soumise à de constants remaniements. Il est de même des impacts résultant des perturbations des écosystèmes sur les dynamiques socio-économiques.

\section{Impacts écologiques}

7 Une communauté scientifique impliquée dans l'étude des écosystèmes anthropisés, s'est constituée en réseau autour de l'élaboration de la notion de résilience ${ }^{10}$. Une autre accentue ses efforts sur l'étude des relations des dynamiques socio-économique et environnementale. Elle forge et documente la notion de vulnérabilité des sociétés humaines ${ }^{11}$. Leur rapprochement actuel est motivé par l'idée qu'il doit être possible de mettre à profit l'usage du concept de résilience dans l'analyse socio-économique, complément indispensable à celui de vulnérabilité, et que réciproquement les 
écosystèmes présentent des formes de vulnérabilité qui contrastent avec leur capacité de résilience. L'apparent détour par ces problématiques nous conduit au cœur de la question du refuge écologique.

En effet s'il est difficile de comprendre le fonctionnement d'un écosystème, il est encore plus malaisé de prévoir les impacts écologiques d'une catastrophe d'autant qu'elle peut soit affecter momentanément l'équilibre du système, soit en affecter le fonctionnement plus ou moins durablement, voire en changer l'organisation structurelle ${ }^{12}$.

9 L'occurrence d'une catastrophe ne laisse place qu'à une action de réparation des dommages et d'endiguement des effets indirectement induits (sur la santé, l'ordre public, la disponibilité et l'accessibilité des ressources, etc.). Idéalement cette action se déploie sur plusieurs temps : l'urgence pour les premiers secours, le moyen terme pour la réparation et le long terme pour restituer au système socio-environnemental une viabilité durable.

Divers observateurs ont relevé la nécessité de la mise en place de mesures préventives. De telles mesures ne peuvent être structurées que face à un risque identifié. Le risque est le produit d'un aléa - par exemple d'origine géophysique, climatique, biologique, industriel - et d'une vulnérabilité sociétale ou environnementale. Divers organismes procèdent à l'analyse des catastrophes ${ }^{13}$ passées afin d'associer aux risques encourus des fréquences probabilistes d'occurrence et des évaluations des dommages potentiels. Par exemple l'analyse de la sismicité historique locale conduit à associer une probabilité à l'occurrence de tremblements de terre en fonction de leur magnitude dans une région donnée. L'analyse des infrastructures urbaines et de la qualité des habitats différencie ensuite la vulnérabilité des sites menacés puis, en considération des aléas, permet d'évaluer les risques avérés. De manière similaire les impacts majeurs d'un cyclone de la classe de Katrina avaient été annoncés par des universitaires américains, en relation notamment avec l'occupation des sols et les infrastructures de gestion des bassins versants en Louisiane (Marris 2005). Ces travaux montrent l'opportunité de prendre des mesures préventives.

11 Avec une fréquence estimée d'environ un événement similaire tous les 500 ans, le tsunami de décembre 2004 a surpris par son ampleur même les experts en gestion des risques. Une telle catastrophe ressort plutôt de la catégorie des risques potentiels : risques identifiés mais auquel on ne peut attribuer de manière fiable une fréquence d'occurrence. Leurs impacts sociétaux et économiques sont d'autant plus importants qu'il est difficile de les prévenir ou mitiger par des mesures idoines.

12 Corrélativement la pression exercée a posteriori par les victimes (individus ou institutions) de tels évènements sur les assurances toucherait aux limites de capacité du système international fondé sur une large mutualisation des portefeuilles de risques (Godard et al., 2002). Par ailleurs, la complexité de fonctionnement des écosystèmes, la diversité des interdépendances entre sociétés et environnements et la dimension aléatoire et complexe des risques écologiques conduit à supposer l'existence d'une catégorie de risques omis, latents mais totalement ignorés à ce jour. Cette hypothèse raisonnable, confortée par quelques catastrophes, en particulier sanitaires, récentes, conduit l'industrie de l'assurance à réfléchir aux mesures à mettre en œuvre pour faire face aux "inconnues inconnues ${ }^{14}$ ». Un même souci anime divers gouvernements qui cherchent à évaluer les coûts et dommages potentiels des crises environnementales en 
opposant une stratégie de non intervention à des scenarii développant des mesures de prévention ou de « mitigation $»^{15}$ (voir Stern, 2006 pour le changement climatique).

\section{Incidences sociétales}

13 Face à la diversité des catastrophes et des risques écologiques ou environnementaux, se déploie une série d'incidences sociétales multidimensionnelles. De nombreux travaux montrent qu'à l'échelle mondiale, la vulnérabilité des sociétés aux catastrophes augmente très significativement depuis quelques décennies (Schiermeier 2005). Deux facteurs principaux, qu'une utilisation à fin préventive ou de progrès technologiques n'équilibrent pas, expliquent cette tendance: la croissance démographique et l'augmentation de la pression d'occupation dans les zones à risque, notamment les zones littorales ou inondables.

14 Le développement de systèmes d'alerte précoce et de surveillance, l'amélioration des habitats, l'ancrage d'une culture du risque, la mise en place de services d'urgence, permettent de réduire le nombre de victimes et les dommages occasionnés notamment lors de catastrophes naturelles ${ }^{16}$ dans les pays ayant la capacité financière de développer une politique préventive. Inversement de nouveaux indicateurs promus par le Programme de Développement des Nations unies (UNDP 2004) montrent une très forte corrélation entre le niveau de développement d'un pays et le taux de mortalité associé aux catastrophes naturelles. Ces taux varient d'un facteur 100 à 1000, selon le type de catastrophe, entre populations riches et populations pauvres. L'estimation du coût des dommages matériels et de leur incidence économique reflète surtout le niveau des investissements dont a été dotée une région touchée par un désastre. On peut supposer qu'une région économiquement développée aura plus de moyens de réparer les dommages subis et de relancer son activité. Ainsi, le coût des dommages causés par un cyclone touchant les côtes nord américaines du Golfe du Mexique sera bien supérieur à ceux d'un cyclone de même classe traversant l'Amérique Centrale. Cependant ce dernier génèrera des ruptures irréversibles des dynamiques économiques, sociales et politiques (d'Ans 2005), handicaps supplémentaires à un développement équilibré.

La logique de l'enchaînement des causes et conséquences est similaire pour des catastrophes plus diffuses. Lorsque plusieurs années consécutives fortement déficitaires en précipitations ont touché le Sahel, les divers groupes sociaux des zones rurales ont été contraints de développer de nouvelles stratégies de survie, tentant de sauver leurs vies, leurs moyens de subsistance, puis leurs droits et leurs institutions (Thebaud, 2002; Raynaut, 1997). L'une d'elles, la migration, est à son tour source de conflits. En milieu rural la rareté des ressources n'explique pas seule ces conflits, d'autres considérations, morales ou politiques par exemple, interviennent aussi (Turner 2004).

Abrupte ou lente, spatialement focalisée ou diffuse, réversible ou irréversible, toute catastrophe écologique est susceptible d'induire une migration massive ${ }^{17}$. Cependant, le niveau de vie très bas des populations touchées est une condition déterminante de la migration de masse. L'analyse de la pauvreté conduite par Dubois et al. (2001) permet de la décliner sous diverses dimensions sociétales. En ne considérant que les deux premières de ces dimensions - économique et environnementale - la "pauvreté d'accessibilité » recouvre déjà les manques d'accès à l'emploi et au crédit, les défauts de 
revenus et de consommation, le non accès aux ressources naturelles, à l'eau, aux semences. La " pauvreté de potentialité » traduit l'absence de capital physique (habitat, foncier,...) financier et naturel (terre de culture, bétail, rareté des ressources hydriques). Face à des conditions de vie trop précaires que vient encore bouleverser une catastrophe, les potentialités que représente la migration pour un groupe social d'accéder à une vie meilleure emportent la décision. Cette orientation est encore appuyée par les autres dimensions sociales (accès limité à la santé, à l'éducation ; ruptures des liens sociaux, etc.), culturelles, politiques et éthiques de la pauvreté (Dubois et al., 2001).

17 Il apparaît clairement que les incidences sociétales d'une catastrophe environnementale d'envergure dépendent des conditions de vie des populations victimes. De plus ces incidences sont fortement interdépendantes : l'organisation du tissu social, des réseaux économiques, des conditions sanitaires et de santé, de l'accessibilité aux ressources, etc., induit en retour de nouvelles incidences à tous les niveaux d'organisation de la société et de l'environnement. Ici les effets dominos sont la règle, non l'exception. La stratégie de migration - toujours contrainte, parfois forcée - résulte d'un faisceau de mobiles et de causes. Le terme de réfugié écologique ou de réfugié environnemental ne peut que couvrir une large classe de situations concrètes, hybrides de déterminants environnementaux mais aussi socioéconomiques ${ }^{18}$. Aussi lorsque les populations sont durement touchées, les bases sociales du développement économique sont bouleversées mais aussi le tissu social lui-même. C'est toute une classe d'âge d'enfants et d'adolescents qui se trouve privée de famille après le tsunami. Une telle situation contraint les conditions d'accueil et de prise en charge des victimes à l'échelle de temps d'une génération au moins.

Quelle est la situation aujourd'hui? On estime que plus d'un être humain sur deux habite dans une zone où la probabilité de l'occurrence d'une ou plusieurs catastrophes naturelles ${ }^{19}$ est élevée. Relativement à d'autres facteurs, l'évolution démographique et ses disparités entre continents ou régions sont assez bien prévues. Cette croissance se double d'une concentration des populations dans des zones à risques, notamment les zones littorales. Les estimations donnent $80 \%$ de la population mondiale vivant dans des pays en développement et $60 \%$ de celle-ci exposée à des risques d'inondation ou de séisme en 2050 (Dauphiné, 2003). La superposition des cartes des zones soumises à des aléas récurrents avec celles de forte vulnérabilité permet une première régionalisation des zones d'émigration potentielle. D'autres facteurs s'avèrent aussi déterminants pour la prise de décision collective d'une migration de masse. La prévision des destinations, magnitude, date et caractère (temporaire, pendulaire ou définitive) des migrations potentielles semble hors d'atteinte (Döös, 1997) sauf cas particuliers. Ainsi, la montée du niveau des océans et l'impact sur la production alimentairetous deux induits par le réchauffement du climat, provoqueraient à eux seuls la migration de l'ordre de 150 millions de personnes d'ici l'horizon 2050. Cette estimation présentée avec une grande prudence par Norman Myers (2003) du fait de sa nature très exploratoire et révisable, n'a cessé d'être reprise et critiquée. Le fait est qu'elle pourrait bien être supérieure à la réalité mais aussi bien inférieure. Mais vers où se dirigeront ces migrations, sachant que les zones littorales offrent de nombreuses ressources?

19 De même, les relations internationales qui ont conditionné les itinéraires migratoires entre États africains lors de la sécheresse des années 1983-1987 (Mattsson and Rapp 1991), ont elles-mêmes grandement évolué. Or la dynamique des migrations 
environnementales dépend des politiques mises en œuvre pour répondre à la demande des réfugiés mais également des solidarités existantes au sein de groupes dotés d'une identité affirmée. Cependant aujourd'hui, aucune protection spécifique des réfugiés écologiques n'existe.

\section{Incapacités et inadaptations du droit pour protéger les réfugiés écologiques}

Différentes causes expliquent cette carence. Tout d'abord, la nature même du droit international public sur lequel est construit le droit international des réfugiés constitue un obstacle à une protection globale des réfugiés écologiques. Ensuite, le droit international humanitaire traditionnellement protecteur des victimes de conflits armés, tente, en vain, d'orienter sa protection vers les victimes de catastrophes écologiques. Enfin, le droit international de l'environnement, qui s'est considérablement enrichi ces dernières années, mais ne s'est pas encore intéressé à toutes les questions humaines, pourtant, cruciales comme celles des flux migratoires liés aux catastrophes environnementales.

\section{Inadaptations structurelles du droit international public et du droit international des réfugiés}

Les migrations liées aux catastrophes écologiques suscitent et susciteront plusieurs sortes de déplacements à l'intérieur et vers l'extérieur des États touchés. Envisager une protection internationale des réfugiés écologiques requiert de garantir des droits aux personnes déplacées à l'intérieur de l'État et à celles qui migrent en dehors de l'État. Autrement dit, une protection qui dépasserait les frontières territoriales des États ${ }^{20}$ (ces dernières n'ayant pas grand sens lors de graves catastrophes écologiques étant donné que leurs impacts complexes touchent souvent plusieurs États). Pourtant cette protection absolue, sans limitation rationae loci du réfugié écologique, semble prématurée au regard des capacités actuelles du droit international. Plusieurs raisons à cela : la nature même du droit international des réfugiés et celle du droit international.

D'abord, une personne menacée qui quitte son pays d'origine peut être protégée par le droit international des réfugiés, essentiellement régi par la Convention de Genève. Mais celle-ci ne protège pas les personnes victimes de catastrophes écologiques (Magniny, 1999 : 56-95 ; Cournil, 2006). La Convention de Genève souffre d'importantes limites ${ }^{21}$ tant sur le champ de sa protection que sur l'instrument lui-même particulièrement

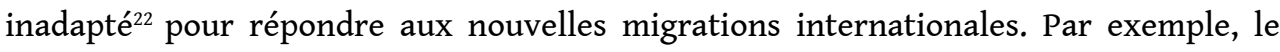
droit international des réfugiés est basé sur une approche individualisée des motifs d'exil du réfugié. Or l'examen individuel effectué par les autorités compétentes de l'État d'accueil paraîtrait inapproprié pour le refuge écologique. En effet, les personnes victimes de catastrophes écologiques fuient en grand nombre le lieu du drame. L'approche prima facie (collective, par groupe) paraît plus adéquate face aux situations d'urgence. De plus, la Convention de Genève est fortement marquée par la nature interétatique du droit international et le respect des souverainetés nationales. Ainsi, un État accorde la protection internationale seulement aux personnes ayant quitté leur pays, c'est-à-dire uniquement celles qui n'ont plus de protection étatique. Dès lors, les personnes déplacées à l'intérieur de leur pays d'origine en raison d'une catastrophe ne 
relèvent pas du champ d'application de la Convention de Genève, et ce, même si le pays touché ne peut leur apporter de protection effective. En effet, le principe de noningérence dans les affaires de l'État et le respect de l'intégrité territoriale empêche la protection des «réfugiés internes ». Il existe donc une différence importante entre les réfugiés conventionnels et les réfugiés écologiques : «les premiers ne bénéficient plus de la protection juridique de leur État d'origine ou de nationalité, alors que pour les seconds, le lien juridique avec leur État n'est pas rompu mais temporairement inopérant » [sauf cas extrême]. Le réfugié conventionnel a besoin d'un statut palliant la rupture juridique avec son pays d'origine "alors que les réfugiés de l'environnement nécessitent un statut atténuant la déficience matérielle de leur État " sans qu'il faille, nécessairement dans tous les cas, remplacer un lien juridique (Magniny, 1999: 462). En somme, le droit des réfugiés paraitt inadapté face aux nouvelles migrations notamment celles liées aux catastrophes écologiques.

Ensuite, même si le droit international connait une évolution certaine en reconnaissant l'individu, il demeure principalement un droit interétatique (Dailler et Pellet, 1999 : 37). Le respect de la souveraineté des États demeure son principe fondateur. Ainsi, le droit international s'adresse aux États, principaux sujets de droit. Or, un droit international protégeant les victimes de catastrophes écologiques devrait pouvoir s'affranchir de certains principes de ce droit interétatique, notamment celui du principe de noningérence ${ }^{23}$ et du principe de l'intégrité territoriale afin de devenir un véritable droit humain, un droit jus cogens (impératif à l'égard de tous, sans condition de réciprocité) ${ }^{24}$. En effet, les impacts écologiques peuvent être d'une telle ampleur que des migrations internes ont lieu sans que l'État touché puisse apporter de solution efficace. Il serait alors souhaitable que la victime déplacée dans son pays obtienne par exemple une protection internationale. Mais, le principe d'égalité des États ${ }^{25}$, du respect des souverainetés et celui de la non-intervention ne permettent théoriquement pas de s'occuper des affaires internes d'un pays même défaillant. Or, pour une partie de la doctrine, les droits humains sont exclus de ce domaine réservé à la compétence nationale, on pourrait tout à fait imaginer que l'assistance aux déplacés internes victimes d'une catastrophe écologiques relève de la protection des droits internationaux des droits de l'Homme, organisée dans le cadre d'un «droit d'ingérence » humanitaire ou écologique (Bachelet, 1995). En effet, certaines ONG et une partie de la doctrine revendiquent ce droit d'ingérence ou d'intervention humanitaire pour secourir des personnes en détresse (cf. infra).

Les défis soulevés par la gestion des futures migrations internationales nous amènent à souligner les inadaptations du droit international tel qu'il a été bâti jusqu'ici. Les conséquences en terme de flux migratoires des prochaines catastrophes écologiques doivent nous conduire à penser autrement le droit international des réfugiés.

\section{Les insuffisances du droit international humanitaire face aux catastrophes écologiques}

Le droit international a, assez récemment, tenté d'apporter des solutions ponctuelles aux victimes (migrantes ou non) de certaines catastrophes écologiques par le biais du droit international humanitaire (DIH). Le DIH au sens classique ne s'adresse qu'aux victimes de conflits armés et ne s'applique donc pas aux victimes de catastrophes écologiques (Bélanger, 2002: 55-79). Toutefois, depuis le début des années quatre- 
vingt $^{26}$, des organisations non gouvernementales interviennent sur les lieux de catastrophes écologiques, en proclamant leur "droit d'ingérence humanitaire ", sorte de «droit à l'assistance internationale humanitaire " (Bettati, 1994). Dépassant le principe du respect des souverainetés nationales et son corollaire le «droit de nonintervention", ce "droit d'ingérence humanitaire" a posé le socle minimal d'une assistance internationale aux victimes de catastrophes. Ce « droit » d'ingérence, que les ONG et une partie de la doctrine juridique ont longtemps réclamé, a soulevé d'intenses débats (Bettati, 1996 : 119 et Harouel-Bureloup 2005 : 499). Adoptée sous l'impulsion de la France, une première Résolution $n^{\circ} 43 / 131$ du 8 décembre $1988^{27}$ de l'Assemblée Générale des Nations unies a fondé les bases de ce droit international humanitaire "moderne ${ }^{28}$. Cette résolution a affirmé le "principe du libre accès aux victimes de catastrophes naturelles et autres situations d'urgence $"{ }^{29}$. Peu de temps après, une seconde résolution $n^{\circ} 45 / 100$ du 14 décembre $1990^{30}$, a créé les couloirs humanitaires afin de permettre le libre acheminement de l'assistance à travers le territoire de l'État sinistré. Ces couloirs humanitaires ont été inspirés du $"$ droit de passage innocent $»^{31}$ dans les eaux territoriales d'un État riverain. Ces résolutions ${ }^{32}$ encouragent les États victimes de catastrophes à faciliter l'acheminement des secours humanitaires (approvisionnement en eaux, vivres, médicaments, campements, etc.). Elles ont « légalisé » l'action des ONG en permettant aux populations qui ne peuvent être secourues par leurs propres pouvoirs publics de recevoir une aide internationale. Avec ces résolutions, «la notion de droit d'assistance humanitaire est devenue, en quelque sorte, l'une des dimensions opérationnelles de la garantie des droits de l'Homme » (Bettati, 1996 : 112).

Toutefois, ces résolutions ne vont pas très loin dans leurs exigences puisqu'elles ne proposent qu'une offre de secours que l'État peut accepter ou non selon la situation d'urgence ${ }^{33}$. Certes, les multiples résolutions adoptées ont permis d'instaurer les bases minimales de l'assistance humanitaire. Néanmoins, si ces deux résolutions ont crée beaucoup d'espoir, ce DIH n'amène pas encore de solutions satisfaisantes pour les ONG spécialisées. D'une part, parce que ces textes reconnaissent toujours le principe du respect des souverainetés nationales et donc le rôle prioritaire des pays victimes dans l'organisation des secours à leurs ressortissants, ce qui gêne l'affirmation d'un droit à assistance internationale autonome. D'autre part, parce que ce droit n'est pas suffisamment contraignant, il a une force obligatoire limitée. Simples recommandations, les résolutions ne sont pas assimilées à un véritable droit ${ }^{34}$. Les résolutions ne font qu'inviter les États à faciliter la mise en œuvre de l'apport de nourriture, de médicaments ou de soins médicaux pour lequel l'accès est indispensable. De plus, ces résolutions ne prévoient aucune sanction pour l'État qui refuse de porter secours à sa population ou qui récuse une assistance extérieure. En somme, ce soft law particulièrement consensuel dans ces objectifs maintient, côte à côte, le respect des souverainetés et le droit d'ingérence humanitaire. La proclamation d'un véritable droit humain impératif donc contraignant, celui d'un droit à la vie, à l'assistance de personnes menacées, n'est pas encore affirmé. Ainsi, force est de reconnaître qu'il n'existe aucun régime international contraignant régissant l'assistance humanitaire aux victimes de catastrophes écologiques (Hardcastle et Chua, 1998). Il n'y a pas d'équivalent à la Convention de Genève de 1949 relative aux victimes de conflits armés, pour les victimes de catastrophes écologiques. Aucun traité multilatéral ne fixe un véritable droit pour ces victimes à recevoir une aide humanitaire. Au regard de ces multiples réflexions, on ne peut que constater la faiblesse des obligations internationales en matière d'assistance aux victimes de catastrophes naturelles. 
Depuis la fin des années soixante, le droit international de l'environnement (DIE) a réglementé et encadré les activités humaines relatives à l'environnement à travers de nombreux textes internationaux ${ }^{36}$ nés d'une prise de conscience des multiples détériorations de l'environnement. Les efforts nationaux ne pouvant plus ou pas répondre aux impacts complexes des nombreuses dégradations anthropiques planétaires, le passage de l'activité normative nationale à internationale est apparu assez vite une évidente nécessité (Kiss et Beurier, 2004: 13). Seule une coopération internationale semblait à la hauteur du défi de la protection de l'écosphère. Ainsi, tout comme la protection des réfugiés écologiques déplacés à l'intérieur ou à l'extérieur des États, la protection de la planète nécessite des normes et institutions internationales. Le droit international de l'environnement (DIE) est très vaste (Romi et al., 2005), il est assez délicat de délimiter son champ d'action. Il se construit très rapidement et par étapes. Ainsi, les années soixante-dix ont été marquées par une protection sectorielle de l'environnement (eau, air, vie sauvage, etc.). Puis, le droit international a réglementé les substances dangereuses et depuis les années quatre-vingt-dix, il s'est engagé vers « une protection intégrée de l'environnement, prenant en compte le développement et le contexte économique mondial. [...] Un nombre croissant d'instruments juridiques tend à fonder la réglementation protectrice de l'environnement sur les activités humaines avec leurs multiples facettes» (Kiss et Beurier, 2004: 15-16). Le champ de ce droit international est aujourd'hui principalement tourné vers le respect, la protection et la conservation de l'environnement. Il traite également des réglementations des activités humaines dangereuses sur plusieurs éléments de l'environnement (sols, forêts, milieu marin, eaux continentales, atmosphère, climat, vie sauvage, biodiversité, etc.). Par ailleurs, le DIE s'est axé sur l'information des populations sur les risques de dégradations et sur les activités néfastes pour l'écosystème. Les grands principes internationaux de l'environnement (principe de précaution, d'information, de prévention et du pollueurpayeur) montrent que le champ de ce droit est essentiellement orienté vers la prévention des risques futurs ou probables. En revanche, la gestion d'une crise ou d'une situation d'urgence telle qu'une catastrophe écologique aux graves conséquences humaines y est peu développée. En effet, même si le DIE a développé un pan entier du droit de la responsabilité et de l'indemnisation et qu'il pose les règles en matière de responsabilité des dégâts environnementaux sur la planète grâce à un volet civil ou pénal, tout l'aspect du droit de l'assistance et de la gestion de l'urgence face à une crise écologique soudaine ou diffuse s'est peu développé. De même, si l'indemnisation est

Revue européenne des migrations internationales, vol. 23 - $n^{\circ} 1$ | 2007 
possible, aucun statut particulier de victime n'est attribué au plan international. Néanmoins, une partie du DIE a traité du sort des victimes de catastrophes nucléaires. Ainsi, en 1985 et 1986, la directive sur les arrangements relatifs à l'assistance mutuelle d'urgence en cas d'accident nucléaire ou de situation d'urgence radiologique et la Convention sur l'assistance en cas d'accident nucléaire ${ }^{37}$ ont imposé aux États parties le devoir de coopérer entre eux en cas de catastrophe nucléaire. Si ce principe existait implicitement dans les relations internationales, ces textes affirment le devoir de venir en aide aux États victimes de catastrophes nucléaires (Kiss et Beurier, 2004: 399). Toutefois, cette assistance concerne la coopération mutuelle entre États ${ }^{38}$ et ne pose pas de véritables droits et obligations envers les victimes. Ces mesures minimales ne s'adressent qu'à l'organisation et au fonctionnement des pouvoirs publics des États sinistrés sans s'intéresser aux victimes. Dès lors, ce DIE essentiellement sectoriel (victimes de catastrophes nucléaires) ne constitue pas une protection directe des victimes déplacées en raison de catastrophes écologiques. De même, si le principe noncontraignant de Rio pose le devoir d'information ${ }^{39}$ en cas de catastrophe écologique, il n'impose pas d'assistance écologique. L'assistance est prévue au sein de quelques conventions internationales sectorielles (Convention de New York sur le droit d'utilisation des cours d'eau à des fins autres que la navigation dont l'article 28, alinéa 3 et 4 impose la coopération en cas de nécessité). Il en est de même des accords bilatéraux sur les pollutions marines ou des accords d'assistance subrégionaux (Kiss et Beurier, 2004 : 140-142).

Par ailleurs, la déclaration de Stockholm a souligné dans son préambule ${ }^{40}$ les dégâts que peuvent subir les hommes du fait de la dégradation de l'environnement. De cette déclaration émerge le principe du droit à l'environnement ${ }^{41}$. Il sera ensuite proclamé en 1981 pour la première fois dans un traité international, dans la Charte africaine des droits de l'Homme ${ }^{42}$. On retrouve le droit à l'environnement et les obligations qui en découlent pour les États dans de nombreuses conventions internationales. Ainsi, les conditions de vie rendues difficiles par un environnement dégradé sont prises en compte notamment par le biais du «droit à un environnement sain ». Ce droit est issu de la troisième génération des droits de l'Homme, des « droits de solidarité ». S'il a été reconnu au niveau international et constitutionnel par quelques pays et qu'il se développe, peu à peu, devant la Cour européenne des droits de l'Homme ${ }^{43}$, ce droit à un environnement sain n'a pas encore de portée suffisamment opérationnelle pour protéger les réfugiés écologiques. Devant la Cour européenne des droits de l'Homme, il se développe indirectement, "par ricochet ", puisqu'il n'a pas d'existence propre, ni explicite dans le texte de la Convention européenne des droits de l'Homme. C'est donc une construction des juges européens qui permet d'élaborer des jurisprudences innovantes (Sudre, 1997).

31 Par exemple, la protection de l'homme face à son environnement dégradé passe aussi par le droit à la santét4. Le droit à la santé est garanti par de multiples textes internationaux constitutionnels et nationaux (Belaidi, 2004: 90-95): il implique un environnement de qualité. Ce droit à la santé a évolué vers un droit à la qualité de vie et vers un droit à la vie notamment grâce à la jurisprudence dynamique de la Cour européenne des droits de l'Homme, (Marguenaud, 2003). L'État a des obligations positives lorsque des dégradations environnementales entraînent des conséquences sur la santé, la qualité de vie et le droit à la vie des personnes. Toutefois, les systèmes actuels de protection internationale des droits de l'Homme sont loin d'être satisfaisants 
et l'on peut émettre des réserves sur leur portée effective sur les nombreuses victimes d'une catastrophe écologique. De plus, la migration temporaire ou définitive d'un réfugié écologique n'est pas envisagée en tant que telle. Pourtant, elle constitue souvent une des premières conséquences d'une grave dégradation du lieu de vie à la suite d'une catastrophe. Autrement dit, aussi encourageant qu'il soit, le droit de l'Homme à un environnement sain ne peut actuellement pas apporter de réponse à la protection collective des réfugiés écologiques.

32 Face à ces multiples inadaptations et insuffisances du droit pour protéger les réfugiés écologiques, les juristes doivent imaginer et proposer des solutions juridiques permettant de faire face aux nombreuses migrations écologiques.

\section{Les défis du droit pour protéger les réfugiés écologiques}

33 Des propositions de protections à instituer (notamment celle d'une protection internationale) ont été émises par la doctrine juridique, que nous rappelons et commentons ci-dessous. Des pistes relatives à des évolutions de concepts juridiques sur une protection des réfugiés écologiques seront ensuite retracées.

\section{Des propositions juridiques à discuter}

Deux propositions de protection sont envisagées : soit la création d'une protection spécifique, soit le renforcement de la protection des personnes déplacées internes déjà existante.

\section{Une protection internationale à créer}

À l'instar de la thèse de Véronique Magniny, l'Appel de Limoges en juin 2005 a énoncé une proposition de protection internationale du « réfugié de l'environnement ». Plusieurs caractéristiques ont été avancées pour définir cette protection; notamment celles développées par Véronique Magniny (dont nous partageons l'analyse) : une protection internationale, autonome, matérielle, collective et juridique (Magniny, 1999 : 491-504).

$\mathrm{Au}$ regard des catastrophes écologiques passées, leur gestion et donc la protection des réfugiés écologiques ne peuvent trouver de réponse adaptée que par la solidarité internationale. Les impacts complexes de nombre de catastrophes écologiques ne permettent plus de raisonner en terme de frontière étatique et donc de protection nationale. De plus, ils ne sont pas seulement environnementaux mais aussi économiques, sociaux et humains. Dès lors, une protection globale donc internationale s'impose $^{45}$. Aussi, Véronique Magniny propose d'opter pour l'adoption d'un traité international multilatéral afin que les États puissent, notamment, être contraints par leurs engagements internationaux. Une Convention permettrait d'entériner les règles internationales coutumières en matière d'accueil des réfugiés, règles d'hospitalité minimales essentiellement développées dans certains pays du Sud (Magniny, 1999: 97-137). Ce serait également l'occasion d'insérer de nouvelles exigences pour l'accueil des réfugiés. Ainsi, en plus des règles coutumières de non-refoulement et d'accueil temporaire, il serait ambitieux d'intégrer dans un traité international des obligations sur le traitement digne des réfugiés par le pays d'accueil, et ce, que le déplacement du 
réfugié soit interne ou international. En plus d'une simple tolérance dans le territoire d'accueil, des droits supplémentaires tenant à l'entretien physique et moral, au droit au travail, au logement décent ainsi que des obligations selon les catégories de victimes pourraient être insérés dans ce texte international. Très ambitieuse, cette proposition aurait certainement aujourd'hui du mal à obtenir le nombre nécessaire de signatures pour son entrée en vigueur. Le contexte actuel n'est pas favorable : les pays du Nord se sont engagés depuis plusieurs années dans des politiques restrictives de l'asile (Dias Urbano de Sousa, 2004) ${ }^{46}$.

37 Par ailleurs, cette protection internationale devrait certainement être collective. Nos analyses ont montré l'inadaptation de la Convention de Genève en raison notamment de son approche individuelle. Les dégâts des catastrophes écologiques brutales ou insidieuses entraînent la plupart du temps des migrations de groupes (villages, villes ou régions détruites, etc.). L'approche prima facie sera sans doute préférable pour protéger au plan international les réfugiés écologiques. La fin de la protection internationale devra se faire aussi pour l'ensemble du groupe. Ce qui peut d'ailleurs poser des problèmes lorsque de vastes régions d'un même pays ont été touchées différemment ou que des zones plus sensibles retrouvent moins rapidement leur équilibre écologique. L'approche collective de la migration risque d'être la caractéristique la plus difficile à accepter pour les États du Nord, et ce, même si les États membres de l'Union européenne ont récemment adopté une directive instituant une protection temporaire collective $^{47}$.

Il paraît souhaitable que la protection internationale passe par la création d'un instrument autonome, spécifique et non par un simple amendement ${ }^{48}$ de la Convention de Genève qui est inadaptée. Si un nouveau motif était spécifiquement créé pour prendre en compte les «persécutions écologiques » dans la Convention de Genève, la protection conventionnelle ne s'adresserait qu'aux personnes ayant quitté leur pays d'origine et exclurait les « personnes déplacées » dans leur propre pays. De plus, l'autonomie d'une telle convention internationale aurait le mérite de ne pas entretenir de confusion avec la protection bien spécifique des réfugiés politiques. Néanmoins, si un tel instrument devait être imaginé, sa conception et réalisation ambitieuses pourraient s'avérer très longue à rédiger et à adopter ${ }^{49}$. Et ce d'autant plus, qu'une protection internationale pour les réfugiés écologiques devrait pouvoir instaurer un véritable statut de droit. Des droits et des obligations définis dans un statut juridique devraient leur être reconnus. Ainsi, le principe de non-refoulement, le droit au regroupement familial, au travail, aux prestations sociales des États accueillants devraient être insérés dans le traité international. De plus, des droits élémentaires à la personne humaine (droit à la vie, à la dignité humaine et à la santé) seraient inévitablement reconnus et mis en œuvre dans les pays d'accueil. La protection internationale devrait également pouvoir mettre en place les bases d'une logistique importante, sans quoi cette protection ne serait pas effective, voire théorique. Des installations de camps temporaires devraient pouvoir être instaurées (Magniny, 1999: 503). La protection internationale aura en effet pour objectif premier de satisfaire aux besoins vitaux des populations réfugiées (hygiène, alimentation, logement, soin, etc.). Un fond permanent spécifique serait donc nécessaire pour que les personnes déplacées puissent reconstruire provisoirement ou définitivement leur vie après la catastrophe écologique. C'est en partie pour cela que le fonds central d'urgence humanitaire a été crée le 15 décembre 2005 par une résolution de l'Assemblée générale des Nations unies. Il devrait permettre aux agences onusiennes d'intervenir plus rapidement lors des catastrophes humanitaires (donc écologiques). Il 
serait souhaitable que ce fonds sollicite massivement les États à contribuer pour la protection spécifique des réfugiés écologiques.

Par ailleurs, Véronique Magniny estime que la protection internationale du réfugié écologique devra être temporaire (moins longue qu'une génération; Magniny, 1999 : 504). Ainsi, dès lors que le retour (sans danger) dans le pays sinistré est possible, la protection internationale s'arrêtera pour l'ensemble du groupe qui a été reconnu réfugié écologique. Le pays d'accueil et le pays sinistré mettront alors en place des programmes de réinstallation. Toutefois, si la protection temporaire peut parfaitement s'envisager pour des catastrophes aux dégâts écologiques de court terme, on peut s'interroger sur la durée de la protection des victimes de dégâts irréversibles ou quasiirréversibles. Ainsi, ceux occasionnés par une catastrophe nucléaire comme Tchernobyl sont tels qu'une réinstallation sur des terres non-contaminées ne peut pas se faire avant une à plusieurs générations. De même, des déplacements définitifs peuvent avoir lieu lors de choix technologiques ou de développement voulus par un État (même si ce ne sont pas à proprement parler des catastrophes). Par exemple, la construction d'un barrage (Trois Gorges en Chine) a entraîné des déplacements définitifs puisque les habitants de la région ont été contraints d'abandonner leurs terres. En somme, lorsque les dommages sont irréversibles, les migrations deviennent définitives, la protection internationale ne peut pas être temporaire. C'est pourquoi il est peut-être préférable que la protection internationale du réfugié écologique n'ait pas de limites ratione temporis afin qu'elle "s'adapte aux catastrophes ", c'est-à-dire qu'elle cesse selon les circonstances et qu'une analyse objective, au cas par cas, détermine la fin de la protection. Elle cesserait, par exemple, lorsqu'il existerait une possibilité de réinstallation, la fin des risques de contamination ou encore une possibilité de reprendre une vie normale. De surcroît, lorsqu'il s'agit des populations étrangères ayant migré en raison d'une catastrophe écologique aux dégâts irréversibles, il convient, peut-être, d'organiser au sein des États accueillants, une sorte de passerelle vers le droit commun des étrangers. Le pays d'accueil doit pouvoir lui offrir une carte de séjour lui permettant de s'intégrer dans les meilleures conditions possibles (avec possibilité de demander la nationalité du pays d'accueil ${ }^{50}$ après des années de résidence selon les mécanismes légaux en vigueur). Par ailleurs, lorsque des réfugiés écologiques fuient définitivement et en grand nombre leur pays sinistré, il serait souhaitable que les États les plus voisins aient l'obligation de les accueillir. Aujourd'hui par exemple, les habitants de l'île Tuvalu ont demandé l'aide de la Nouvelle-Zélande et de l'Australie pour être accueillis ${ }^{51}$, lîle devant peu à peu disparaitre sous la montée des eaux. La protection de onze mille habitants de Tuvalu devrait pouvoir être organisée avant la disparition de lîle, "par prévention". La protection internationale du réfugié écologique pourrait alors imposer ce type obligation aux États voisins ${ }^{52}$. Ces derniers actuellement repliés sur leur souveraineté étatique se préservent aujourd'hui des flux migratoires. Pourtant ici, on est loin d'être dans le cas d'une «immigration économique " tant redoutée par les pays du Nord, il s'agit bien d'une immigration forcée imposée par les changements climatiques. L'affaire Tuvalu apparaît, aujourd'hui encore, comme un cas d'école ${ }^{53}$, mais la mise en place d'une protection du réfugié écologique permettrait d'imposer des obligations internationales en matière d'hospitalité, d'assistance et de solidarité. 


\section{Renforcement de la protection des personnes déplacées internes}

40 sont de plus en plus nombreuses ${ }^{54}$. De multiples causes expliquent ces déplacements internes : guerres ethniques intra-étatiques, guerres civiles, catastrophes écologiques ${ }^{55}$, famines, etc. Certaines de ces " personnes déplacées internes » migrent en raison d'une cause écologique (catastrophes naturelles, déplacées en raison de projet de développement, etc). Aussi, la notion de PDI pourrait constituer la base d'une protection englobante affirmée par le droit international (et de ce fait inclure les réfugiés écologiques).

Depuis 1994, la doctrine internationale, les organisations internationales ${ }^{56}$, les organisations non gouvernementales ${ }^{57}$ ont engagé un travail de systématisation et de définition de la notion de PDI. Ainsi en 1998, Francis Deng ${ }^{58}$ a proposé une définition juridique $^{59}$ de la PDI dans un document intitulé "principes directeurs relatifs au déplacement de personnes à l'intérieur de leur propre pays $»^{60}$. Selon ces principes, "les personnes déplacées à l'intérieur de leur propre pays jouissent, sur un pied d'égalité, en vertu du droit international et du droit interne, des mêmes droits et libertés que le reste de la population $d u$ pays $»^{61}$ et l'assistance des PDI doit être assurée par les autorités nationales ${ }^{62}$ (Mooney, 2005: 11). Dès lors, les déplacés internes en raison d'une catastrophe écologique sont avant tout théoriquement protégées par leur État. Ces principes ont été reconnus comme une norme utile de Soft Law, ils n'ont aucun effet contraignant et souffrent par conséquent d'une application effective réduite dans la plupart des pays concernés. Aussi, depuis l'affirmation de ces principes, la doctrine, les organisations internationales et les organisations non gouvernementales ont présenté de nombreux projets $^{63}$ et propositions afin que des garanties complémentaires plus opérationnelles soient intégrées dans le droit interne et la pratique des États. Par exemple, selon un projet sur "le déplacement de personne à l'intérieur de leur propre pays ${ }^{64}$, l'État doit s'acquitter de sa responsabilité en respectant 12 points ${ }^{65}$ essentiels envers les déplacés. La doctrine encourage même les États à intégrer dans leur droit interne ${ }^{66}$ (affirmation des droits) et à mettre en pratique (application effective) les principes directeurs et certaines bonnes pratiques d'accueil (liberté de passage pour les ONG, sécurité du personnel humanitaire, etc.). Ce travail doctrinal permet surtout de proposer un véritable cadre pré-normatif aux États afin de leur rappeler leurs obligations et responsabilités. L'apport essentiel des réflexions doctrinales engagées depuis 1998 a été d'établir une liaison cruciale entre d'une part les déplacements internes et d'autre part le droit international des droits l'Homme et le droit international humanitaire.

Depuis les années soixante-dix et surtout depuis l'affirmation des principes directeurs, le HCR a entrepris des collaborations informelles avec les États en matière d'assistance et de protection des déplacés. Il a également coopéré avec d'autres institutions des Nations unies en leur faveur (évaluation des besoins et planification de l'assistance) ${ }^{67}$. En 2005, le rôle du HCR a été sensiblement clarifié et encouragé afin qu'il assure la coordination et la gestion des camps de déplacés et des refuges d'urgence (Cohen, 2005 : 9-11). Jusqu'à présent, aucun organisme des Nations unies n'était spécifiquement chargé des personnes déplacées. De ce fait, c'est par une action concertée des agences des Nations unies et des ONG que ces personnes étaient secourues. Mais l'action concertée s'est parfois révélée inefficace voire catastrophique (notamment au Darfour, au Libéria) du fait de nombreux facteurs: dilution des responsabilités des multiples acteurs de terrain, flou dans le partage des compétences et rivalités entre les 
organismes compétents (Eschenbächer 2005 : 15-16 et Charny 2005 : 20-21). Dès lors, les nouvelles compétences du HCR parviendront-elles à assurer une protection et une assistance intégrative des PDI et notamment de celles qui fuient une catastrophe écologique?

En définitive, la notion de PDI et le travail doctrinal qui l'entoure sont pertinents pour l'assistance et la protection des victimes de catastrophes écologiques. Néanmoins, cette assistance n'en est qu'à ces balbutiements ; d'autres règles minimales complémentaires devraient pouvoir leur être encore affirmées et garanties, telles que le droit à l'assistance et à la non-discrimination de l'aide (de manière à éviter le détournement de l'aide par des groupes, des minorités, une caste etc.), la protection spécifique des femmes et des enfants, la sécurité publique des déplacés (protection des camps), l'accès à un logement, à un niveau de vie convenable (emploi et accès aux terres), au soin, à l'éducation, la facilitation des démarches administratives (papiers d'identité, titres fonciers, etc.), une bonne gestion des droits de propriété après les catastrophes écologiques, la libre circulation dans le pays, l'intégration locale, la possibilité d'envisager un retour raisonnable ou une re-installation dans d'autres régions, etc... Il faut espérer que les récents drames écologiques vont accélérer la mise en place de normes plus claires et permettre une véritable protection des réfugiés écologiques...

\section{Des concepts juridiques à inventer}

L'urgence de futurs mouvements de populations en raison d'une cause écologique nous pousse à réfléchir à des concepts juridiques capables de faire avancer la recherche juridique sur une protection internationale du réfugié écologique. Quatre pistes peuvent être creusées: celle de «l'ingérence écologique», de la notion "d'État défaillant », de la « force majeure » et du « droit d'asile environnemental ».

L'idée d'ingérence écologique a été notamment développée par Michel Bachelet (1995). Selon lui, la protection de la planète doit passer par des normes multisectorielles, des normes de solidarités internationales afin de faire face, voire de prévenir les risques majeurs que court la planète. Ainsi, ces normes pourraient passer par un abandon partiel de la souveraineté étatique au profit d'une nouvelle façon de régler le comportement des relations entre États. Il relève avec justesse que l'assistance aux victimes de catastrophes naturelles ou anthropiques existe mais sous forme de pratiques empiriques sectorielles sans réelle unité entre elles $^{68}$. Sorte de «droit à l'assistance humanitaire " en matière environnementale, l'ingérence écologique permettrait, au nom de la solidarité internationale, de dépasser les limites du principe de souveraineté qui empêche toute intervention obligatoire dans le pays sinistré. Comme les grandes catastrophes naturelles ont des effets planétaires, la communauté internationale justifierait d'un droit d'ingérence dans les affaires des États qui polluent ou subissent un drame écologique aux impacts transnationaux (par ex. les pollutions transfrontalières). En effet, face aux risques majeurs, il y a " une incompatibilité entre les frontières étatiques et les données naturelles de la planète » (Bachelet, 1995 : 214).

La protection du réfugié écologique pourrait constituer un exemple pratique d'un nouvel ordre humanitaire international en mettant en œuvre l'idée d'ingérence écologique. Celle-ci permettrait aux victimes laissées dans la détresse d'un drame écologique en raison des carences ou défaillances de l'État de revendiquer un droit d'assistance et de protection juridique. L'ingérence écologique poserait les bases d'une 
proclamation d'un droit international des droits de l'Homme relatif à la protection des victimes de catastrophes écologiques. L'accès et l'assistance aux victimes ne seraient plus constitués comme une "infraction » mais comme une "intervention curative " ${ }^{69}$, une «assistance écologique " (Lavieille, 2006). A l'instar de Mario Bettati, force est de reconnaître que l'argument du respect des souverainetés étatiques lors de catastrophes écologiques est "anachronique». Il souligne également que l'adoption des grands textes internationaux relatifs aux droits fondamentaux témoigne d'une évidente évolution du droit international. Le droit des victimes de catastrophes écologiques doit émerger dans ce contexte où deux logiques internationales contradictoires s'affrontent: le droit international classique et le droit international des droits de l'Homme. Toutefois, Michel Bachelet rappelle avec raison que l'ingérence (quelle qu'elle soit) sera difficile sans " une autorité réellement supranationale capable d'imposer ses décisions au niveau de leur exécution effective " (Bachelet, 1995 : 214 ${ }^{70}$ ). La création d'une institution internationale spécialisée permettrait d'encadrer les interventions et d'être la gardienne unique des motifs d'intervention. Or l'instauration de cet organe supranational est subordonnée à la reconnaissance d'un droit impératif général ou jus cogens.

Une autre notion sur laquelle des réflexions doivent également être engagées est celle de "l'État défaillant». La défaillance à assurer une protection aux victimes de catastrophes écologiques peut se révéler à la fois dans l'action comme dans l'inaction de l'État. Soit l'État agit et prend des mesures pour protéger ces ressortissants mais celles-ci se révèlent insuffisantes ou inadaptées. Soit l'État n'engage pas de protection spécifique et laisse dans la détresse ces ressortissants (l'exemple de la pollution d'un fleuve chinois causée par l'explosion d'une usine de benzène en novembre 2005 a montré que le gouvernement chinois a caché à la population touchée la pollution pendant près de dix jours. Il a néanmoins porté secours à la population après coup). Cette notion pourrait également permettre la mise en œuvre d'une nouvelle responsabilité internationale (Michelot-Draft, 2006 : 428-445).

Le concept de force majeure pourrait constituer une piste de réflexion et servir de critère objectif de déclenchement de la protection du réfugié écologique. En effet, la notion de force majeure est habituellement utilisée pour exonérer l'administration d'une responsabilité pour faute ou responsabilité pour risque (en droit français). Or ici, le concept pourrait s'utiliser non pas comme une cause d'exonération mais comme une cause de déclenchement d'une protection. Ainsi, en disposant d'un ou de plusieurs critères objectifs (telle que la force majeure) une institution internationale pourrait déterminer si la situation écologique nécessite une protection. En France, la notion de force majeure est strictement définie par le juge ${ }^{71}$. On retient traditionnellement une définition en trois points : le caractère extérieur aux victimes, l'imprévisibilité (dans sa survenance) et l'irrésistibilité (dans ses effets) de l'événement. La force majeure suscitée par une catastrophe doit être d'une violence exceptionnelle. Néanmoins, si cette notion pouvait servir à distinguer les "simples victimes" des réfugiés écologiques, elle aurait sans doute le handicap de ne pas inclure certaines victimes de catastrophes plus insidieuses (par exemple la sécheresse) dont la violence n'est pas aussi «visible» que l'impact d'un tremblement de terre ou d'une importante inondation. En tous les cas, la force majeure procèderait à une qualification juridique des faits, concrète, et ce en tenant compte de toutes les circonstances de la catastrophe. L'institution chargée de la qualification des faits pourrait développer sa propre 
jurisprudence à condition que la Convention internationale qui l'instituerait ait des critères suffisamment précis et clairs de définition de la force majeure.

Un «droit d'asile environnemental» a été proposé par un parti d'opposition ${ }^{72}$ en Australie le 5 janvier 2006. Il s'est exprimé en faveur d'un "droit d'asile environnemental» pour les populations des petits États insulaires océaniens (Papouasie Nouvelle-Guinée, îles Marshall, Kiribati, Tuvalu, États Fédérés de Micronésie etc.), dont les atolls sont directement menacés par la montée du niveau de l'océan Pacifique. Cette proposition vise à inciter le gouvernement australien à élaborer une véritable politique d'accueil prioritaire pour ces populations océaniennes menacées. Cet accueil permettrait d'organiser une sorte de "protection anticipatrice" des déplacements massifs de personnes menacées par la montée du niveau des océans. Cette protection inédite, organisée avant la crise écologique, éviterait ou réduirait les risques d'atteintes à la sécurité régionale (notamment en Australie et en Nouvelle Zélande). Si cette proposition a été extrêmement contestée par les autorités australiennes, l'idée d'un "droit d'asile environnemental» est sans doute à creuser, voire à construire pour les premiers « réfugiés du changement climatique ». Celui-ci pourrait être pensé comme une protection offerte par un État voisin et négocié préalablement dans un accord bilatéral d'assistance par exemple. Ce «droit d'asile environnemental » apparaîtrait comme une manifestation d'une solidarité d'un État à l'égard d'un État voisin menacé de disparaître. La proposition de l'opposition australienne insiste sur un « droit d'asile environnemental » limité aux ressortissants de pays insulaires du Pacifique, pays prédéterminés dans une liste. Avec la constitution de cette liste, l'État d'accueil se déclarerait prêt à accueillir d'éventuels réfugiés écologiques venus de pays directement touchés par la montée des océans. D'autres mesures organisant par exemple le séjour dans le pays d'accueil pourrait être imaginées pour mettre en place une version opérationnelle de ce nouveau type de droit (« droit d'asile environnemental »).

50 La protection des réfugiés écologiques bouscule les principes traditionnels du droit international. Un des défis majeurs du xxI ${ }^{\text {ème }}$ siècle sera sans aucun doute de parvenir à dépasser ces paradigmes trop étroits pour envisager une protection véritablement opérationnelle.

\section{BIBLIOGRAPHIE}

AEN Agence pour l'énergie nucléaire (2002) Tchernobyl - Évaluation de l'impact radiologique et sanitaire, (mise à jour 2002 du document « Tchernobyl : Dix ans déjà), 173 p.

APPEL de LIMOGES sur les réfugiés écologiques du 23 juin 2005 (http://www.cidce.org/).

BACHELET Michel (1995) L'ingérence écologique, éd. Frison-Roche, 304 p.

BELAIDI Nadia (2004) La lutte contre les atteintes globales à l'environnement vers un ordre public écologique, Thèse (Dact.) Université des de Bourgogne, 612 p.

BELANGER Michel (2002) Droit international humanitaire, éd. Gaulino, Paris, 150 p. 
BETTATI Mario (1994) Intervention ingérence ou assistance ?, Revue trimestrielle des droits de l'Homme, $\mathrm{n}^{\circ}$ 19, pp. 307-358.

BETTATI Mario (1996) Le droit d'ingérence, mutation de l'ordre international, éd. Odile Jacob, 384 p.

BOUTEILLET-PAQUET Daphné (2002) La protection subsidiaire : progrès ou recul du droit d'asile en Europe ? Une analyse critique de la législation des États membres de l'Union européenne, in Daphné Bouteillet-Paquet Dir., La protection subsidiaire des réfugiés dans l'Union européenne : un complément à la Convention de Genève ? Bruylant, Bruxelles, pp. 181 et s.

Brookings Institution-Université de Berne (2005) Projet sur le déplacement de personnes à l'intérieur de leur propre pays, « Faire face au problème du déplacement de personnes à l'intérieur de leur propre pays : cadre normatif précisant les responsabilités des États ", 41 p. (http:// www.brookings.edu/fp/projects/idp/idp.htm)

CHARNY Joël R. (2005) La nécessité d'une nouvelle approche face aux déplacements internes, Revue des Migrations Forcées, pp. 20-21.

COHEN Roberta (2005) L'élargissement du rôle du HCR aux personnes déplacées à l'intérieur de leur pays, Revue des Migrations Forcées, pp. 9-11.

CORTEN Olivier et KLEIN Pierre (1992) L'assistance humanitaire face à la souveraineté des États, Revue trimestrielle des droits de l'Homme, $\mathrm{n}^{\circ}$ 11, juillet, pp. 343-364.

COURNIL Christel (2005) Le statut interne de l'étranger et les normes supranationales, éd. L'Harmattan Collection Logiques Juridiques, $744 \mathrm{p}$.

COURNIL Christel (2006) Vers une reconnaissance du « réfugié écologique » Quelle(s) protection(s) ? Quel(s) statut(s) ?, Revue du droit public, juillet-août 2006, n 4, pp. 1035-1066.

COURNIL Christel et MAZZEGA Pierre (2006) Catastrophes écologiques et flux migratoires : Comment protéger les « réfugiés écologiques » ?, Revue européenne de droit de l'environnement, décembre $\mathrm{n}^{\circ}$ 4, pp. 417-427.

D’ANS André-Marcel (2005) Écologie politique d'un désastre - Le Honduras après l'ouragan Mitch, ed. Karthala, Paris, 280 p.

DAILLER Patrick et PELLET Alain (1999) Droit international public, éd. LGDJ, 7ème éd., Paris, 1510 p.

DAUPHINE André (2003) Risques et catastrophes, observer, spatialiser, comprendre, gérer, éd. Armand Colin, Paris, $288 \mathrm{p}$.

DE RAULIN Arnaud (2006) Gouvernance et urgence, in Arnaud De Raulin dir., Situation d'urgence et Droits fondamentaux, Paris, pp. 149-163.

DIAS URBANO DE SOUSA Constança (2004) L'émergence d'une politique européenne de l'asile, éd. Bruylant, Bruxelles, 352 p.

DÖOS Bo R. (1997) Can large-scale environmental migrations be predicted? Global Env. Change, 7(1), pp. 41- 61.

DUBOIS J-L., MAHIEU F.R. et A. POUSSARD (2001) La durabilité sociale comme composante du développement humain durable, in Thiébaut $\mathrm{D}$. Dir., Développement : vers un nouveau paradigme, ed., cahiers du GRATICE n 20, Univ. Paris XII, France, pp. 95-110.

EL-HINNAWI E., Environmental refugees, PNUE, 1985, Nairobi, 41 p.

ESCHENBÄCHER Jens-Hagen (2005) Comment assurer un meilleur fonctionnement du système d'action concertée, Revue des Migrations Forcées, décembre, pp. 15-16. 
GEISLER Charles and DE SOUSA Ragendra (2000) From refuge to refugee: the african case, Working paper $\mathrm{N}^{\circ} 38$, Land Tenure Center, Univ. of Wisconsin, Madison (USA), 19 p.

GODARD Olivier, HENRY Claude, LAGADEC Patrick et MICHEL-KERJAN Erwann (2002) Traité des nouveaux risques : Précaution, crise, assurance, Gallimard / Folio actuel, 620 p.

GONIN Patrick et LASSAILLY-JACOB Véronique (2002) Les réfugiés de l'environnement, une nouvelle catégorie de migrants forcés ?, Revue Européenne des migrations Internationales, (18) 2, pp. 139-160.

GOUGUET Jean-Jacques (2006) Réfugiés écologiques : un débat controversé, Revue européenne de droit de l'environnement, décembre $\mathrm{n}^{\circ}$ 4, pp. 381-399.

HARDCASTLE Rohan J. et. CHUA Adrian T. L. (1998) Assistance humanitaire : pour un défi à l'accès aux victimes des catastrophes naturelles, Revue internationale de la Croix-Rouge, $\mathrm{n}^{\circ}$ 832, pp. 633-655.

HAROUEL-BURELOUP Véronique (2005) Traité de droit humanitaire, Presse Universitaire de France, $499 \mathrm{p}$.

HAUT COMMISSARIAT DES NATIONS UNIES POUR LES REFUGIES (1997) Les réfugiés dans le monde. Les personnes déplacées : l'urgence humanitaire, éditions La Découverte, 297 p.

JULIENNE M. (2002) 25 millions de réfugiés écologiques, Québec Science, juin.

KISS Alexandre et BEURIER Jean-Pierre (2004) Droit international de l'environnement, éd. Pedone, études internationales, $503 \mathrm{p}$.

LASSAILLY-JACOB Véronique (2006) Une nouvelle catégorie de réfugiés en débat, Revue européenne de droit de l'environnement, décembre $n^{\circ}$ 4, pp. 374-380.

LAURENT C. (2003) Un droit à la vie en matière environnementale reconnu et conforté par une interprétation évolutive du droit des biens pour les habitants de bidonvilles, Revue trimestrielle des droits de l'Homme n 53, pp. 261-297.

LAVIEILLE Jean-Marc (2006) L'assistance écologique, Revue européenne de droit de l'environnement, décembre $\mathrm{n}^{\circ} 4$, pp. 400-412.

MAGNINY Véronique (1999) Les réfugiés de l'environnement, hypothèse juridique à propos d'une menace écologique, Thèse de droit (dact.), Université de droit, Paris, Panthéon Sorbonne, 645 p.

MARGUENAUD J.-P. (2003) Droit de l'Homme à l'environnement et Cour européenne des droits de l'homme, Revue juridique de l'environnement, $\mathrm{n}^{\circ}$ spécial, La charte constitutionnelle en débat, septembre 2003, pp. 15-21.

MARRIS Emma (2005) The vanishing coast, Nature, vol. 438, n 7070, pp. 908-909.

MATTSSON Jan O. and Anders RAPP (1991) The recent droughts in western Ethiopia and Sudan in a Climatic context, AMBIO, 20 (5), pp. 172-175.

MICHELOT-DRAFT Agnès (2006) Enjeux de la reconnaissance du statut de réfugié écologique pour la construction d'une nouvelle responsabilité internationale, Revue européenne de droit de l'environnement, décembre $\mathrm{n}^{\circ} 4$, pp. 428-445.

MOONEY Erin (2005) Responsabilité étatique et déplacement internes : cadre d'intervention, Revue des Migrations Forcées, décembre, pp. 11-14.

MYERS Norman (1993) Environmental refugees in a globally warmed world, Bioscience, 43, pp. 752-761. 
RAYNAUT Claude (Dir.) (1997) Sahels - Diversité et dynamiques des relations sociétés-nature, ed. Karthala, Paris, 430 p.

ROMI Raphaël, BOSSIS Gaëlle, ROUSSEAUX Sandrine (2005) Droit international et européen de l'environnement, Montchrestien, Paris, $368 \mathrm{p}$.

SANDOZ Y. (1992) Droit ou devoir d'ingérence, droit d'assistance : de quoi parle-t-on ?", Revue internationale de la Croix-Rouge, $\mathrm{n}^{\circ}$ 795, mai-juin, pp. 225-237.

SCHIERMEIER Quirin (2005) The chaos to come, Nature, vol. 438, n 7070, pp. 903-906.

STERN Nicholas (2006) Stern Review Report on the Economics of Climate Change, Cambridge Univ. Press, London, in press.

SUDRE Frédéric (1997) La protection du droit à l'environnement par la Convention européenne des droits de l'Homme, in Jean-Claude Masclet Dir., La communauté européenne et l'environnement, Colloque d'Angers, la Documentation française, Paris, pp. 211-212.

THEBAUD Brigitte (2002) Foncier pastoral et gestion de l'espace au Sahel, Karthala, Paris, 320 p.

TURNER Matthew D. (2004) Political ecology and moral dimension of "resource conflicts": the case of farmer-herder conflicts in Sahel, Political Geography, 23, pp. 863-889.

UNITED NATIONS DEVELOPMENT PROGRAMME (2004) Reducing disaster risk - A challenge for development (Global Report), Bureau for Crisis Prevention and Recovery, http://www.undp.org/ bcpr, 146 p.

\section{NOTES}

1. Flux prévisionnel estimé à près de 150 millions de personnes pour 2050 (Myers, 1993).

2. Ch. Cournil a bénéficié d'une bourse post-doctorale du Centre National d'Études Spatiales. This work was performed within the framework of the AMMA project. Based on a French initiative, AMMA has been constructed by an international group and is currently funded by large number of agencies, especially from France, the UK, the US and Africa. It has been the beneficiary of a major financial contribution from the European Communities Sixth Framework Research Programme. Detailed information on the scientific coordination and funding is available on the AMMA international web site (https://www.amma-eu.org/).

3. Selon le Rapport de 1985 du Programme des Nations Unies pour l'Environnement (PNUE) «les réfugiés environnementaux sont des personnes forcées de quitter leurs habitations traditionnelles d'une façon temporaire ou permanente à cause (naturelle ou humaine) d'unedégradation nette de leur environnement qui bouleverse gravement leur cadre de vie et/ qui déséquilibre sérieusement leur qualité de vie». (El-Hinnawi, 1985). "Réfugiés écologiques», «migrants» ou « réfugiés de l'environnement ", " réfugiés climatiques ", "éco-réfugiés ", " personnes déplacées en raison d'une catastrophe naturelle ", " exodes écologiques », etc., beaucoup de termes traduisent l'exil, la migration ou le déplacement en raison d'une atteinte à l'environnement. Toutefois, si nous nommons dans notre article "réfugiés écologiques » les personnes qui fuient leur habitation en raison d'une dégradation de leur environnement, il ne faut pas les confondre avec les réfugiés au sens du Haut Commissariat aux Réfugiés de l'ONU, qui ont fui leur pays d'origine et migré dans un autre pays. Nous englobons aussi ceux qui ont trouvé asile dans leur propre pays, les «personnes déplacées » dans le sens du HCR.

4. Convention de Genève relative au statut de réfugié, adoptée le 28 juillet 1951.

5. Déclaration Universelle des Droits de l'Homme, (ONU), 10 décembre 1948. Convention de sauvegarde des droits de l'Homme et des libertés fondamentales du 4 novembre 1950. Pactes 
internationaux du 19 décembre 1966, relatif aux droits civils et politiques et aux droits économiques, sociaux et culturels, publié par décret n 81-77, 29 janvier 1981, JORF, 1er février 1981. Directive 2004/83/CE du Conseil, du 29 avril 2004, concernant les normes minimales relatives aux conditions que doivent remplir les ressortissants des pays tiers ou les apatrides pour pouvoir prétendre au statut de réfugié ou les personnes qui pour d'autres raisons, ont besoin d'une protection internationale, et relatives au contenu de ces statuts, JOUE, 30 septembre 2004, L 304/ 12, etc.

6. Cour européenne des droits de l'Homme, Comité des droits de l'Homme.

7. L'étude comparée de Daphné Bouteillet-Paquet (2002) a montré que seuls quatre pays membres (Grèce, Suède, Finlande et Italie) prévoyaient un mécanisme de protection dans leur législation pour les personnes fuyant une catastrophe écologique. Seul le Danemark aurait véritablement et explicitement abordé le problème du refuge écologique au moment de Tchernobyl, les instances ayant traité la question mais les requérants n'ayant obtenu ni reconnaissance de la qualité de réfugié, ni titre de séjour pour motifs humanitaires.

8. Directive 2001/55/CE du Conseil, du 20 juillet 2001, relative à des normes minimales pour l'octroi d'une protection temporaire en cas d'afflux massif de personnes déplacées et à des mesures tendant à assurer un équilibre entre les efforts consentis par les États membres pour accueillir ces personnes et supporter les conséquences de cet accueil, JOCE L 212, 7 août 2001, p. 12-23.

9. Appel de Limoges sur les réfugiés écologiques du 23 juin 2005 (http://www.cidce.org/).

10. Consulter le site http://www.resalliance.org/ Nous entendons ici ce terme au sens d'un retour d'un écosystème ou de l'état d'un environnement circonscrit vers un équilibre (dynamique) antérieur après qu'il ait subi une perturbation.

11. Consulter le site http://sust.harvard.edu/

12. Par exemple en changeant la chimie du milieu ambiant et par suite la biocénose, ou bien en supprimant quelques espèces biologiques clefs d'une chaîne trophique, etc.

13. Voir les informations à ce propos rassemblées dans André Dauphiné (2003) et les données du Centre de Recherche sur l'épidémiologie des désastres.

14. Formule du Secrétaire d'État à la Défense D. Rumsfeld : "The challenge for the industry really is to think about the unknown unknowns » cité par Schiermeier 2005.

15. Le terme de mitigation (atténuation) renvoie à la prévention des risques, à l'atténuation des dommages en développant une politique de prévention visant à réduire les risques, la vulnérabilité des milieux voire l'intensité des dégâts causés par les catastrophes afin de les rendre supportables pour la société.

16. Quatre aléas naturels types, tremblements de terre, cyclones, inondations et sécheresses, sont responsables de près de $94 \%$ des décès dus aux catastrophes naturelles.

17. Un cas devrait être considéré à part: les personnes déplacées suite à la création de zones spécifiques de conservation de la biodiversité; ces "réfugiés écologiques » d'un autre type pourraient se compter également par millions (Geisler and de Sousa 2000).

18. Cf. une typologie des départs et des migrations écologiques (Cournil 2006 : 1055).

19. Si les catastrophes d'origine industrielle peuvent avoir de graves incidences sur la santé des populations (par ex. catastrophe de Bhopal de 1984 - Bailly 2004, catastrophe de Tchernobyl de 1986 - AEN 2002, voire inscrire des pathologies génétiques (maladie de Minamata), elles sont plus limitées en nombre de victimes que les catastrophes naturelles. De plus la question du partage des responsabilités se pose de manière plus aiguë pour les premières.

20. Véronique Magniny (1999: 491) insiste sur le dépassement de la fiction juridique de la frontière au profit d'une conception pragmatique.

21. La Convention de Genève souffre surtout de l'interprétation restrictive des autorités nationales chargées de reconnaitre la qualité de réfugié.

22. En effet, ce traité a été signé après la Seconde Guerre Mondiale dans un contexte migratoire différent de celui d'aujourd'hui. Depuis une vingtaine d'années, la dislocation des États-Nations, l'augmentation de la pauvreté, la multiplication des guerres civiles et ethniques ainsi que 
l'augmentation de l'occurrence des catastrophes écologiques ont changé le contexte migratoire. Les migrations internationales ont évolué et se sont diversifiées. Progressivement de nouvelles causes d'exil sont apparues. Face à ces nouvelles migrations, le droit international des réfugiés est en crise.

23. "L'ingérence désigne en droit international l'immixtion sans titre d'un État ou d'une organisation intergouvernementale dans les affaires qui relèvent de la compétence exclusive d'un État tiers ", (Bettati, 1996 : 12). Ce principe est présent à l'article $2 \S 7$ de la Charte des Nations-Unies.

24. Les droits de l'Homme sont considérés par une partie de la doctrine comme impératif par nature. La notion de norme impérative de droit international général est définie par la Convention de Vienne du 23 mai 1969, dans son article 53 : « Aux fins de la présente Convention, une norme impérative de droit international général est une norme acceptée et reconnue par la communauté internationale des États dans son ensemble en tant que norme à laquelle aucune dérogation n'est permise et qui ne peut être modifiée que par une nouvelle norme du droit international général ayant le même caractère".

25. « Les États sont égaux juridiquement entre eux [...]. Le droit international est réducteur et négateur des différences réelles entre États. Ne permettant pas, la plupart du temps, de prévenir ou de corriger les inégalités de dimension, de richesse, de puissance, il constitue un obstacle à toutes les tentatives pour faire consacrer juridiquement une typologie inégalitaire des statuts des États. [...] L'égalité souveraine peut conduire à négliger et à perpétuer des inégalités concrètes entre États ", (Dailler et Pellet, 1999: 425-426).

26. La Croix Rouge italienne (suivant l'initiative de Giovanina Ciraolo) avait dès 1921 réfléchi à des protections pour les victimes de calamités publiques. De même en 1922, un projet de statut d'une œuvre internationale de secours et d'assistance aux populations victimes de calamités a été proposé. Puis en 1927, la SDN a crée le bureau international de secours, etc. Plus d'informations (Harouel-Bureloup, 2005 : 500).

27. Résolution $n^{\circ} 43 / 131$ adoptée par l'Assemblée générale des Nations-Unies, le 8 décembre 1988 relative à l'assistance humanitaire aux victimes des catastrophes naturelles et situation d'urgence du même ordre.

28. Appellation d'une partie de la doctrine du droit international humanitaire.

29. $\S 4$ de la Résolution $n^{\circ} 43$ : «L'accès aux victimes ne saurait être entravé ni par l'État touché ni par un État voisin ».

30. Résolution $n^{\circ} 45 / 100$ adoptée par l'Assemblée Générale des Nations-Unies, le 14 décembre 1990 relative à l'assistance humanitaire aux victimes des catastrophes naturelles et situation d'urgence du même ordre.

31. Article 17 de la convention de Montego Bay sur le droit de la mer.

32. Les deux résolutions ont été complétées, le 3 février 1995, par la Résolution n 49/139 sur le renforcement de la coordination de l'aide humanitaire d'urgence fournie par l'ONU.

33. L'urgence est mise en avant par une partie de la doctrine pour justifier un droit d'exception, erga omnes, sorte de gouvernance internationale pour légitimer les interventions humanitaires (De Raulin, 2006).

34. Voir Corten et Klein, 1992 : 343-364 ; Sandoz, 1992.

35. "Profondément préoccupée par la souffrance des victimes de catastrophes naturelles et situation d'urgence du même ordre, par la perte en vies humaines, les destructions de biens et les déplacements massifs de populations qui en résultent ", Résolution n 43/131, adoptée par l'Assemblée des NationsUnies, le 8 décembre 1988 relative à l'assistance humanitaire aux victimes des catastrophes naturelles et situation d'urgence du même ordre.

36. Conférence des Nations unies sur l'environnement (Stockholm, juin 1972); 1er Sommet de la terre (Rio de Janeiro 3-14 juin 1992); Convention-cadre des Nations unies sur les changements climatiques (1992) ; Protocole à la Convention sur le climat dit Protocole de36-Kyoto (adopté en décembre 1997, et entré en vigueur le 16 février 2005) ; 4ème conférence de l'ONU sur le climat 
(Buenos Aires, 2-14 novembre 1998); Sommet mondial sur le développement durable (6-17 décembre 2004) ; 10ème Conférence des Nations unies sur les changements climatiques (Buenos Aires, 8 janvier 2005), etc.

37. Adoptée à Vienne, 26 septembre 1986.

38. Article 2 sur la fourniture d'assistance entre les États parties.

39. Déclaration de Rio sur l'environnement et le développement : cf. principes 10 et 19.

40. $\S 3$ de la Déclaration de Stockholm : « [...] Aujourd'hui, ce pouvoir qu'a l'homme de transformer le milieu dans lequel il vit, s'il est utilisé avec discernement, peut apporter à tous les peuples les bienfaits du développement et la possibilité d'améliorer la qualité de la vie. Utilisé abusivement ou inconsidérément, ce même pouvoir peut causer un mal incalculable aux êtres humains et à l'environnement. [...] Des perturbations profondes et regrettables de l'équilibre écologique de la biosphère; la destruction et l'épuisement de ressources irremplaçables; enfin de graves déficiences qui sont dangereuses pour la santé physique, mentale et sociale de l'homme, dans l'environnement qu'il crée, et en particulier dans son milieu de vie et de travail. [...] Principe 9, Les déficiences de l'environnement imputables à des conditions de sousdéveloppement et à des catastrophes naturelles posent des problèmes graves [...] ». Cf. aussi Principe 18 de la Déclaration de Rio, 1992.

41. $1^{\mathrm{er}}$ principe de la Déclaration.

42. Article 24 «Tous les peuples ont droit à un environnement satisfaisant et global propice à leur développement".

43. Cf. par le biais de la protection de la vie privée (article 8 de la CEDH) pour des cas de pollution. Cf. des exemples devant la Cour européenne : CEDH, 21 février 1990, Powel et Rayner, $c$. Royaume-Uni, série A n ${ }^{\circ} 172$; CEDH, 2 octobre 2001, Hatton et autres c./ Royaume-Uni; CEDH, 9 décembre 1994, Lopez-Ostra, série A n 303 ; CEDH, 9 juin 1998, Mc Ginley et Egan c./ Royaume-Uni ; CEDH, 19 février 1998, Anna Maria Guerra et autres c./ Italie. Cf. www.dhcour.coe.fr. Cf. un « droit à la vie » en matière d'environnement dans l'arrêt de la CEDH 30 novembre 2004 (Grande Chambre) Öneryildiz c./ Turquie (requête no 48939/99) (Laurent 2003) et une obligation positive de protection du droit à la vie : CEDH, LCB c/ Royaume-Uni, 9 juin 1998 (essais nucléaires au large de l'île Christmas). Notons plus globalement qu'une motion pour une Recommandation de l'Assemblée Parlementaire du Conseil de l'Europe a été proposée par quelques parlementaires sur la question des réfugiés de l'environnement: "The problem of environmental refugees ", du 23 octobre 2006: http://assembly.coe.int/Main.asp?link=/Documents/WorkingDocs/Doc06/ EDOC11084.htm

44. Le droit à la santé est un concept large il comprend notamment le droit d'accès à l'eau potable, le droit à un environnement sain.

45. A l'image de la protection de la biosphère qui ne doit pas souffrir des frontières artificielles posées par le droit, la protection des réfugiés écologiques doit être organisée par l'ensemble de la communauté internationale.

46. En revanche, les pays du Sud auront certainement plus d'intérêts à soutenir un tel projet puisqu'une grande majorité d'entre eux seront touchés plus souvent et plus durement par les prochaines catastrophes écologiques. De plus, les États du Sud sont davantage habitués à traiter les questions de refuge collectif (Amérique centrale et Latine, Afrique) alors que les pays du Nord (européens) sont fidèles à l'approche individuelle de la Convention de Genève.

47. Directive 2001/55/CE du Conseil, du 20 juillet 2001, relative à des normes minimales pour l'octroi d'une protection temporaire en cas d'afflux massif de personnes déplacées et à des mesures tendant à assurer un équilibre entre les efforts consentis par les États membres pour accueillir ces personnes et supporter les conséquences de cet accueil, JOCE L 212, 7 août 2001, p. 12-23.

48. Cette question a été longuement débattue au colloque de Limoges du 23 juin 2005.

49. En effet, si la Convention internationale relative aux droits des enfants a été adoptée et est entrée en vigueur en un temps "record", la Convention internationale sur la protection des droits de tous les travailleurs migrants et les membres de leur famille a été plus longue à entrer 
en vigueur car elle traite de la question sensible de l'immigration (des travailleurs migrants en situation irrégulière). De plus, cette dernière convention n'a été ratifiée que par les pays " générateurs " d'immigration de travail, aucun pays européen ou pays du Nord ne semble être prêt à adopter un traité leur imposant des obligations en matière d'immigration irrégulière. Autrement dit, les exigences ambitieuses d'un traité sur la protection des " réfugiés écologiques » risquent de susciter peu d'enthousiasme auprès des pays du Nord, particulièrement frileux sur les questions migratoires, ce qui est fort regrettable au regard des risques environnementaux possibles.

50. Cf. opération de naturalisation de masse au Mexique et au Kirghizistan.

51. Ils ont demandé à l'Australie (qui a pour le moment refuser tout accueil) et à la NouvelleZélande (qui a accepté un contingent de personnes à accueillir) de pouvoir bénéficier d'un régime d'immigration préférentiel si les grandes marées rendaient la vie sur l'île impossible. L'association Alofa Tuvalu aide les tuvaliens en cherchant des terres d'asile et en réfléchissant à un statut pour les réfugiés environnementaux. Un premier accord a été passé avec la NouvelleZélande.

52. Toutefois, l'argument des pays voisins chargés d'accueillir les victimes de la catastrophe écologique n'est pas aussi évidente et peut être critiqué. En effet, pour quelle raison serait-ce aux pays voisins de supporter les impacts d'un afflux massif d'immigrants écologiques? Quelles incidences faut-il envisager dans d'autres cas que celui de Tuvalu si l'argument de voisinage finissait par l'emporter dans cette affaire et de ce fait faisait jurisprudence ? Par ailleurs, l'idée de «voisinage » est sujette à des interprétations non-exclusivement cartographiques : voisinage culturel, liens traditionnels entre certaines nations, etc.

53. Toutefois, la communauté villageoise de Lateu a été obligée de se déplacer à un point plus élevé des terres, à l'intérieur de l'île Tegua (archipel de Torres, Vanuatu), et ce, en raison de l'élévation du niveau de la mer. Cette initiative fait partie des stratégies d'adaptation possibles face aux incidences futures des changements climatiques. La sensibilisation aux risques et à la vulnérabilité $d u$ village s'est faite grâce aux concours de multiples acteurs locaux et internationaux (différentes administrations locales et internationales, ONG, églises, jeunes et habitants du village): cf. Rapport sur les travaux de l'atelier consacré à l'article 6 de la Convention-cadre sur les changements climatiques dans la région de l'Asie et du Pacifique, 20 octobre 2005. Cf. aussi le cas d'un village d'Alaska, RICHE Pascal, «Shishmaref, le village qui fond ", Libération, mercredi 19 janvier 2005.

54. Le HCR évaluait à $25 a ̀$ 30 millions le nombre de personnes déplacées à l'intérieur de leur pays, dont 16 millions en Afrique (Haut Commissariat des Nations Unies pour les Réfugiés, 1997).

55. Plus d'un million de personnes déplacées à la suite du tsunami de décembre 2004 .

56. Unité inter-agences sur les déplacements internes (UDI) de l'office des Nations Unies pour la Coordination des Affaires Humanitaires (OCHA), le HCR, la Commission des droits de l'homme mais aussi le Programme alimentaire mondial (WFP - World Food Programme), le Fonds des Nations Unies pour l'Enfance (UNICEF), l'Organisation mondiale de la santé (OMS), le Programme des Nations Unies pour le Développement (PNUD), le Haut Commissariat aux Droits de l'Homme, etc.

57. Comité International de la Croix-Rouge (CICR), Fédération internationale des sociétés de la Croix-Rouge et du Croissant-Rouge (FICR).

58. Ancien représentant du Secrétaire général des Nations Unies pour les personnes déplacées, remplacée depuis 2004 par le Professeur Walter Kälin.

59. «Les personnes déplacées sont des personnes ou des groupes de personnes qui ont été forcés ou contraints à fuir ou à quitter leur foyer ou leur lieu de résidence habituel, notamment en raison d'un conflit armé, de situations de violence généralisée, de violations des droits de l'homme ou de catastrophes naturelles ou provoquées par l'homme ou pour en éviter les effets, et qui n'ont pas franchi les frontières 
internationalement reconnues d'un État ». E/CN.4/1998/53/add.2. 11 février 1998 (instrument non contraignant).

60. À la demande des gouvernements conformément à l'Assemblée générale et à la Commission des Droits de l'Homme des Nations-Unies : «Les principes directeurs relatifs au déplacement des personnes à l'intérieur de leur propre pays » ont été adoptés (E/CN.4/1998/53/add.2. 11 février 1998) (instrument juridique non contraignant).

61. Principes 1.

62. Principes 3.

63. Global IDP Project.

64. (Brookings Institution-Université de Berne, 2005).

65. 1) Empêcher les déplacements de personnes et limiter leurs effets indésirables ; 2) Sensibiliser davantage au problème l'ensemble de la population; 3) Recueillir des données sur le nombre et l'état des PDI ; 4) Soutenir une formation concernant les droits des PDI ; 5) Créer un cadre juridique pour protéger les droits des PDI ; Cadre normatif précisant les responsabilités des États ; 6) Formuler une politique nationale concernant le déplacement de personnes à l'intérieur du pays ; 7) Désigner un point focal institutionnel pour les PDI ; 8) Encourager les organismes nationaux de défense des droits de l'homme à intégrer le problème du déplacement de personnes à leur travail ; 9) Assurer la participation des PDI à la prise de décisions ; 10) Soutenir des solutions durables; 11) Affecter des ressources adéquates au problème ; 12) Coopérer avec la communauté internationale lorsque la capacité nationale n'est pas suffisante.

66. L'Angola, le Burundi, la Colombie, le Libéria, le Pérou, les Philippines et le Sri Lanka, ont intégré des références aux principes directeurs dans le droit interne ou dans leurs politiques. De même, certaines lois non conforme aux principes directeurs ont été modifiées en Géorgie. Enfin, la Cour constitutionnelle colombienne a fait intégrer les principes directeurs dans le droit colombien.

67. Le rôle du HCR s'est considérablement adapté aux nouvelles situations d'exil, il n'a toutefois pas de compétence propre pour régler le sort des "réfugiés écologiques ». Grâce à la procédure des «bons offices », il a défini de nouvelles compétences. Résolutions n 2956 (1972) et n 3455 sur les personnes déplacées(1975) et celle $n^{\circ}$ 43/131 (1989) par laquelle l'Assemblée Générale reconnaissait effectivement que le sort des victimes de catastrophes naturelles et de situations d'urgence, qui n'étaient pas des réfugiés, devait être pris en considération par la Communauté internationale. Voir aussi les résolutions sur l'Éthiopie, Résolution $n^{\circ} 39 / 105$, Assistance aux personnes déplacées en Éthiopie du 14 décembre 1984, Résolution $n^{\circ}$ 40/133 du 13 décembre 1985, la Résolution $n^{\circ}$ 41/141 Assistance aux personnes déplacées et aux victimes de catastrophes naturelles en Éthiopie. Résolution $n^{\circ} 38 / 216$ Assistance aux régions victimes de la sécheresse à Djibouti, Éthiopie, Kenya, Ouganda, Somalie, du 20 décembre 1989.

68. "Sorte d'échantillons de droits sectoriels d'assistance humanitaire spécialisée ", (Bachelet, 1995 : 49). Cf. Organismes spécialisés de l'ONU en matière «d'assistance » : UNICEF, HCR, OMS, FAO-PAM, AIEA, OCHA, etc.

69. Dans la Résolution de Paris, il a été proposé de reconnaître dans un même document international, à la fois le droit des victimes à l'assistance humanitaire et l'obligation des États d'y apporter leur contribution (Bettati, 1996: 91). Mario Bettati parle également d'un droit à l'assistance humanitaire très large sans aucune distinction sur les victimes (conflits armés, catastrophes naturelles ou du fait de l'homme), (Bettati, $1996: 92$ ).

70. Et sur la nécessité de créer une juridiction spécialisée, Bachelet 1995 : 269-282.

71. Exemple devant le juge administratif: CE 10 mai 1912 Ambrosini, CE ass. 28 mai 1971, Département du Var c./Entreprise Bec Frères, CE 22 octobre 1971 Ville de Fréjus.

72. Porte-parole Bob Sercombe. 


\section{RÉSUMÉS}

Des migrations majeures issues de catastrophes environnementales sont attendues lors des prochaines décennies. La conception de mesures adaptées de prévention et de réduction des impacts se heurte à l'absence de scenarii migratoires fiables, composant risques environnementaux avec vulnérabilité et résilience sociétale. Ainsi les instruments juridiques de protection des réfugiés écologiques font défaut. Les limites structurelles du droit international des réfugiés, fondé sur le respect de la souveraineté des États et du principe de non-ingérence, ainsi que celles $\mathrm{du}$ droit international humanitaire moderne et du droit international de l'environnement ne permettent pas d'envisager une protection à la hauteur des enjeux. La protection juridique des réfugiés écologiques doit adapter ou inventer de nouveaux concepts et mécanismes juridiques tels que la création d'une protection internationale et le recours aux notions d'ingérence écologique, de droit d'asile environnemental, de force majeure, d'État défaillant.

Prospective thoughts on a legal protection for ecological refugees. Major migrations induced by environmental disasters are expected for the next decades. The design of adequate prevention and mitigation tools comes up against the low reliability of migration scenarii accounting for environmental risks and societal vulnerability and resilience. Thus legal instruments to protect ecological refugees are lacking. The structural limits of the international law based on the respect of State sovereignty and of the principle of non-interference, as well as those limits of the modern humanitarian international law and international environmental law do not allow to construct a protection tied to the real issue. The legal protection of ecological refugees must adapt and invent new legal concepts and mechanisms like the creation of an international protection and the use of notions like the ones of ecological interference, environmental asylum rights, fortuitous event, failed State.

Reflexiones prospectivas sobre una protección jurídica de los refugiados ecológicos. En las próximas décadas se prevén migraciones importantes debidas a catástrofes medioambientales. La concepción de medidas adaptadas de prevención y reducción de sus impactos se choca con la falta de escenarios fiables de migración, que tomen a cuenta tanto a los riesgos medioambientales como a la vulnerabilidad y a la resiliencia de la sociedad. Tampoco existen instrumentos jurídicos de protección de los refugiados ecológicos. Además los límites estructurales del derecho internacional de los refugiados basado en el respeto de la soberanía de los Estados y en el principio de no ingerencia, así como los limites del derecho internacional humanitario moderno y del derecho internacional del medio ambiente no permiten prever una protección a la altura de lo que está en juego. La protección jurídica de los refugiados ecológicos pues, deberá inventar nuevos instrumentos jurídicos y recorrer a nuevos conceptos como ingerencia ecológica, derecho de asilo medioambiental, fuerza mayor, o Estado deficiente.

\section{INDEX}

Mots-clés : aide humanitaire, droit d'asile, droits, écologie, environnement, population déplacée, protection juridique, réfugiés 


\section{AUTEURS}

\section{CHRISTEL COURNIL}

Maître de conférences en droit public, IRIS (ex CRESP), Université Paris 13, UFR Sciences Médecine Biologie Humaine, 74 rue Marcel Cachin, 93017 Bobigny, France. Courriel : christelcournil@yahoo.fr

\section{PIERRE MAZZEGA}

Directeur de recherche CNRS, Laboratoire des Mécanismes et Transferts en Géologie, UMR 5563 CNRS/IRD/Univ. Toulouse III, Observatoire Midi-Pyrénées, 14 avenue E. Belin, 31400 Toulouse. Courriel : Pierre.Mazzega@cnes.fr 